\title{
Quantizing charged magnetic domain walls: Strings on a lattice
}

\author{
H. Eskes, R. Grimberg, W. van Saarloos, and J. Zaanen \\ Institute Lorentz, Leiden University, P.O. Box 9506, NL-2300 RA Leiden, The Netherlands
}

(Received 19 March 1996; revised manuscript received 17 April 1996)

\begin{abstract}
Recent experiments have shown the existence of an ordered stripe phase in the cuprates, suggesting that the nearby metallic phase is best described as a domain-wall fluid. As a minimal model to describe the melting of charged domain walls we introduce quantum lattice strings. This model is related to a generalized quantum spin chain. The string exhibits a rich phase diagram, containing several rough phases with low-lying excitations as well as gapped ordered phases. In this scenario the occurrence of the stripe phase is related to a transition from the quantum-rough (metal) to the classical flat phase. [S0163-1829(96)51526-2]
\end{abstract}

The study of high- $T_{c}$ superconductors has caused a crisis of the conventional paradigm of metal physics, the Fermiliquid theory which insists that the current in metals is carried by quasiparticles with all properties of electrons except that their effective interactions vanish at the Fermi surface. It seems now widely agreed that this theory fails fundamentally in the context of the high- $T_{c}$ cuprates. Indeed, a strong case can be made that any theory starting with a particlelike vacuum is bound to fail. ${ }^{1}$ From a theoretical viewpoint this suggests that the Fermi-liquid fixed point can be unstable and in recent years there have been many investigations aimed at the possible breakdown of Fermi-liquid theory or at identifying new phases of strongly correlated fermions.

The present study of quantum domain walls is motivated by recent compelling experimental evidence ${ }^{2}$ suggesting that nature has indeed chosen to realize a collective phase whose basic ingredient is an intuitively simple and easily identifiable many-particle bound state: a charged domain wall. Microscopically these domain walls in two dimensions consist of holes bound in a linear stringlike fashion, separating antiferromagnetically ordered regions. Across a wall the antiferromagnetic order parameter points in opposite directions (see Fig. 1). Tranquada et al. find a freezing of these domain walls in a so-called striped phase at a doping concentration of $x=1 / 8$, in the middle of the superconducting regime in $\mathrm{La}_{1.48} \mathrm{Nd}_{0.4} \mathrm{Sr}_{0.12} \mathrm{CuO}_{4}{ }^{2}$

These domain walls were found some time ago to be the generic semiclassical (spin $S \rightarrow \infty$ ) mean-field solutions of models for doped Mott-Hubbard insulators with short-range (on-site) Coulomb interactions only. ${ }^{3}$ As is well known, the motion of a hole in an antiferromagnetic background is hampered by the spin order. In domain walls the holes still have room to fluctuate, so that their kinetic energy is low, while at the same time the number of broken (spin) bonds per hole is small. Domain walls also survive in more sophisticated treatments, ${ }^{4}$ and their formation is favored by Coulomb ${ }^{5}$ and electron-phonon interactions. ${ }^{6}$ Domain wall striped phases were found before in two-dimensional, doped $\mathrm{La}_{2} \mathrm{NiO}_{4}{ }^{7}$ where one is most likely approaching the classical limit more closely ${ }^{6}$ than in the cuprates.

As argued in the paper by Tranquada et al., ${ }^{2}$ the occurrence of the striped phase in the cuprate is suggestive of dominating domain wall correlations in the fluid (metallic, superconducting) phases as well. After all, the freezing is explained $^{2}$ in terms of a simple commensuration effect, which is inactive in the absence of charged domain wall correlations. Moreover, the spin fluctuations in the metallic state $^{8}$ closely match the static spin incommensurations in the ordered state. 9

These observations force one to consider the possibility that the metallic state is a fluid of charged domain walls: a phase with strong dynamical hole correlations which are reminiscent of a disordered domain wall network. We argued before $^{10}$ that if the (collective) dynamics of such a domain wall fluid is assumed to be driven by thermal fluctuations, one obtains a natural explanation for the anomalous magnetic dynamics in the metallic state. This analysis indicates that at not too low temperatures there should be a crossover to a low temperature regime where the fluid becomes dominated by quantum fluctuations-obviously, because the ground state is a superconductor and not a striped phase. Virtually all questions concerning the precise nature of this conjectured quantum domain wall fluid are, at present, wide open.

In this paper, we focus on the quantum meandering fluctuations of a single wall on a lattice. It turns out that a single wall displays a surprisingly rich behavior and it appears as the natural building block to describe both the metallic and ordered phases. In developing a model we are guided by several observations. (i) From Hartree-Fock calculations, ${ }^{3}$ it is known that the energy associated with adding or removing a hole from a domain wall is rather high. Hence we consider the number of holes in a wall to be fixed. (ii) The wellknown fact that long wavelength shape fluctuations of fluid interfaces (i.e., capillary waves) and membranes have a low energy, suggests that these are important low energy modes of a domain wall as well. Moreover, mean field calculations indicate that the "stiffness" associated with these modes is small: kinks have a low creation energy, while their tunnel-

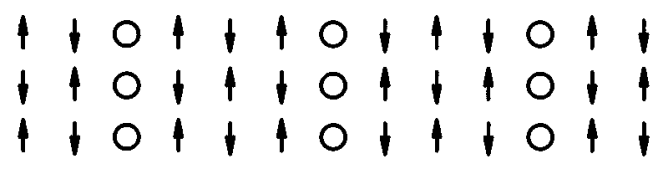

FIG. 1. Schematic illustration of a domain wall of holes separating two antiferromagnetically ordered domains. Note that in reality the hole density may vary more gradually across the wall. 
(a)
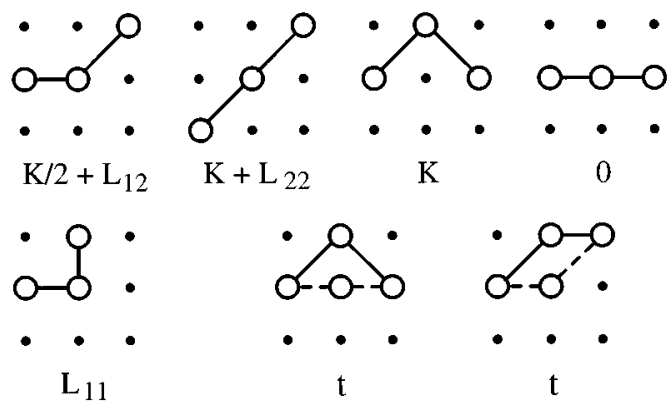

(b)

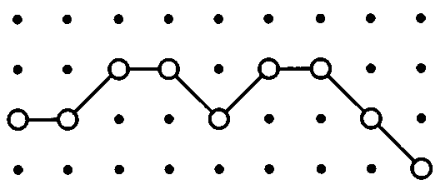

FIG. 2. (a) Energies (of the central hole) and tunneling amplitudes of the various local-bond configurations. The tunneling process is between the dashed configuration and the one drawn in full. (b) Typical configuration of a rough directed string on a square lattice.

ing rate to neighboring sites is appreciable. ${ }^{11}$ (iii) From the theory of interfaces, it is well known that meandering fluctuations are not properly incorporated in a mean field theory, and in order to understand the commensurability effect of a lattice (roughening transition), one has to start with a proper microscopic lattice model. (iv) Quantum mechanical domain wall fluctuations reduce the kinetic energy of a hole. For walls in an antiferromagnetic background (Fig. 1), the low energy fluctuations without spin frustration are those where the holes preserve their stringlike order (as beads on a string); in other words, where the wall does not break up into two disconnected pieces. (v) The latter fluctuations dominate the dynamics of spins near a wall. It is therefore reasonable to neglect in a first stage the interaction between spin waves in the antiferromagnetic domains and the wall degrees of freedom.

The model we propose for the strong hole-binding limit of quantum domain walls is that of quantum lattice strings. These are connected strings of "holes" on a square lattice, successive holes $l$ and $l+1$ having either a distance 1 or $\sqrt{2}$. Let $\left(\eta_{l}^{x}, \eta_{l}^{y}\right)$ be the position of hole $l$. We write the classical interaction as a sum of nearest and next-nearest neighbor discretized string tensions,

$$
\begin{aligned}
\mathcal{H}^{\mathrm{cl}}= & \sum_{l}\left[K \delta\left(\left|\eta_{l+1}^{x}-\eta_{l}^{x}\right|-1\right) \delta\left(\left|\eta_{l+1}^{y}-\eta_{l}^{y}\right|-1\right)\right. \\
& \left.+\sum_{i, j=0}^{2} L_{i j} \delta\left(\left|\eta_{l+2}^{x}-\eta_{l}^{x}\right|-i\right) \delta\left(\left|\eta_{l+2}^{y}-\eta_{l}^{y}\right|-j\right)\right] \\
& +M \sum_{l, m} \delta\left(\eta_{l}^{x}-\eta_{m}^{x}\right) \delta\left(\eta_{l}^{y}-\eta_{m}^{y}\right) .
\end{aligned}
$$

The relevant local configurations are shown in Fig. 2(a). The last term is an excluded volume type interaction; the physically relevant limit is $M \rightarrow \infty$, so that holes cannot occupy the same site ( $L_{00}$ is now irrelevant). We neglect extreme curvature, $L_{10}=\infty$, and choose $L_{20}=0$. Therefore the model is parameterized by $K, L_{11}, L_{21}$, and $L_{22}$. The strings are quantized by introducing conjugate momenta $\pi_{l}^{\alpha}$, $\left[\eta_{l}^{\alpha}, \pi_{m}^{\beta}\right]=i \delta_{l, m} \delta_{\alpha, \beta}$. A term $e^{i n \pi_{l}^{x}}$ will cause hole $l$ to hop a distance $n$ in the $x$ direction. Therefore the hopping contribution in its simplest, nearest-neighbor form ${ }^{12}$ is

$$
\mathcal{H}^{\mathrm{qu}}=2 t \sum_{l, \alpha} P_{\mathrm{str}}^{\alpha}(l) \cos \left(\pi_{l}^{\alpha}\right)
$$

Here $P_{\text {str }}^{\alpha}(l)$ is a projector restricting the motion of hole $l$ in the $\alpha$ direction to string configurations [Fig. 2(a)]. ${ }^{13} \mathrm{By}$ means of the Suzuki-Trotter mapping the quantum problem $\mathcal{H}^{\mathrm{cl}}+\mathcal{H}^{\mathrm{qu}}$ translates to the problem of two coupled $(1+1)$ dimensional restricted solid-on-solid (RSOS) models. The two RSOS height flavors correspond to $\eta_{l, k}^{x}$ and $\eta_{l, k}^{y}, k$ being the imaginary-time Trotter index. The "steps" $\eta_{l+1, k}^{\alpha}$ $-\eta_{l, k}^{\alpha}$ and $\eta_{l, k+1}^{\alpha}-\eta_{l, k}^{\alpha}$ are restricted to $0, \pm 1$.

An important simplification occurs by restricting the allowed configurations to those with $\eta_{l}^{x}=l$. In this directed string problem the bonds between neighboring holes always step to the right [see Fig. 2(b)] — in interface language: the strings have no "overhangs." By using the Suzuki-Trotter or transfer matrix formulation, one can write the ground state problem of a directed lattice string in terms of the statistical mechanics of a classical solid-on-solid surface model. ${ }^{14}$ In this mapping, one may think of the quantum lattice string as tracing out a two-dimensional world sheet in space-time; this formulation has the advantage that it allows a rather natural and direct translation of various phases of the twodimensional surface problem into those of quantum stringse.g., rough surfaces correspond to lattice strings whose quantum fluctuations are so strong that they meander, even in the ground state.

The equivalence between RSOS problems and spin algebras was extensively discussed in the seminal work by den Nijs and Rommelse. ${ }^{14}$ As the links between neighboring holes can only point in three directions [Fig. 2(a)], the Hamiltonian can equivalently be formulated in terms of a spin-1 model, with $S_{l}^{z}=1,0,-1$ corresponding with the link between site $l$ and $l+1$ pointing up, horizontal, and down, respectively. In terms of the string-tension parameters,

$$
\begin{aligned}
\mathcal{H}= & \sum_{l}\left[\left(\frac{L_{22}}{2}-2 L_{21}\right)\left(S_{l}^{z} S_{l-1}^{z}\right)^{2}+\frac{L_{22}}{2} S_{l}^{z} S_{l-1}^{z}\right. \\
& \left.+\left(K+2 L_{21}\right)\left(S_{l}^{z}\right)^{2}+\frac{t}{2}\left(S_{l}^{+} S_{l-1}^{-}+S_{l}^{-} S_{l-1}^{+}\right)\right] .
\end{aligned}
$$

In the special case $L_{22}=4 L_{21}$ the Hamiltonian reduces to the well-known spin-1 chain with single-site anisotropy $D=\left(K+2 L_{21}\right) / t$ and Ising anisotropy $J=L_{22} / 2 t$. The phase diagram as given by den Nijs and Rommelse ${ }^{14}$ is shown in the inset in Fig. 3. However, for $L_{22}>4 L_{21}$ we find several extra phases to occur. Using a combination of quantum Monte Carlo, exact diagonalization, and knowledge of the critical behavior at the various transitions ${ }^{14,15}$ we arrive at the zero-temperature phase diagram for $L_{22} / 2-2 L_{21}=5$ shown in Fig. 3.

There are several ordered phases in Fig. 3. They are listed in Table I. For large negative $L_{22}$ diagonal walls are found 


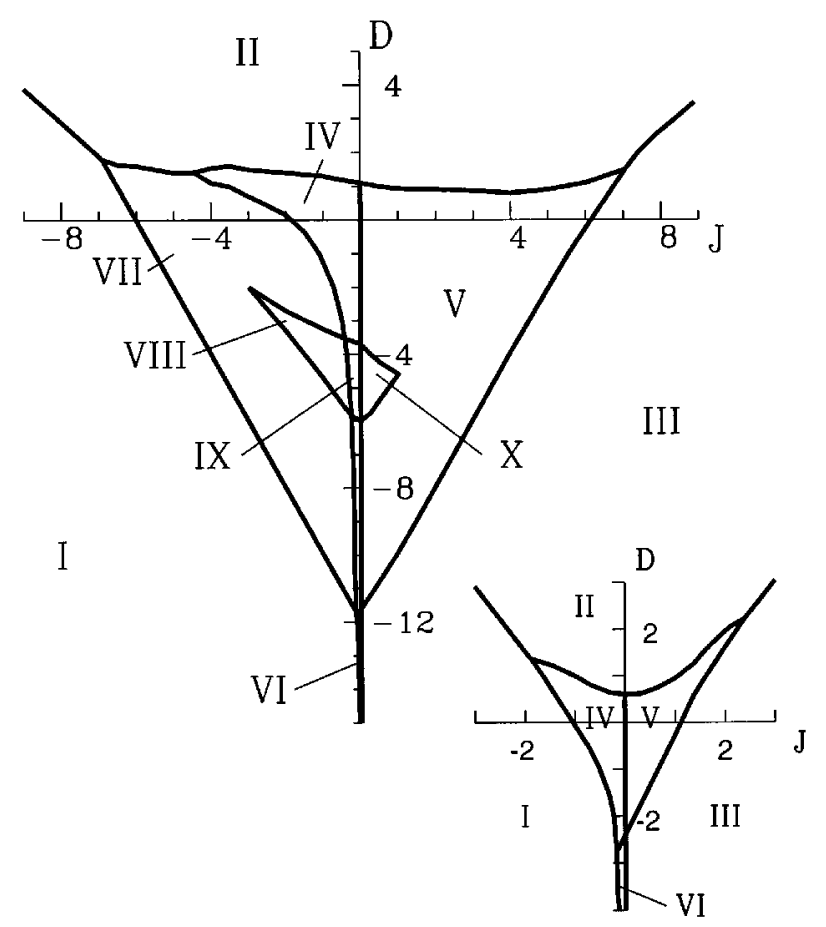

FIG. 3. Phase diagram of the directed lattice-string problem with $L_{22} / 2-2 L_{21}=5$, as a function of $J=L_{22} / 2$ and $D=K+2 L_{21}$. The inset shows the phase diagram for $L_{22}=4 L_{21}$.

(phase I) and for large $K$ the walls are horizontal and flat (phase II). Positive $L_{22}$ (phase III) favors (not very realistic) zigzag walls (or antiferromagnetism in spin language). Apart from these, two new phases occur. Phase VIII corresponds to a $22.5^{\circ}$ slanted phase of alternating diagonal and horizontal bonds (alternating $S^{z}=1$ and $S^{z}=0$ ). Phase $\mathrm{X}$ is similar but now the diagonals themselves have an alternating up step, down step order. The excitation spectra of these phases are gapped. Alternating order of diagonal and horizontal bonds is favored by $L_{22} / 2-2 L_{21}>0$.

The remaining phases are quantum disordered. They can be characterized by the presence and/or absence of order of the diagonal and horizontal bonds. Starting from the flat phase (phase II) and lowering $K\left(L_{22}<0\right)$ the quantum meandering fluctuations become dominant and the lattice string undergoes a roughening transition. ${ }^{16}$ Like rough twodimensional interfaces, the vertical displacements $\eta_{l}^{y}$ of the hole have logarithmically diverging spatial correlations $\left\langle\left(\eta_{l}^{y}-\eta_{m}^{y}\right)^{2}\right\rangle \sim \ln |l-m|$. These rough strings have low lying excitations (capillary waves in interface language, spin waves in spin language). In phase IV both the diagonal and horizontal bonds are disordered. Phase VI occurs at large negative $K$. Here only virtual pairs of horizontal bonds can occur. The model can be mapped on the anisotropic Heisenberg spin-1/2 $X X Z$ model and phase VI corresponds to an anisotropy parameter $-1<\Delta<1$ of this model. Phase $\mathrm{V}$ is the disordered flat or Haldane phase. In this region up steps of the strings are followed by down steps, but the location of these up and down steps has no long range order because the horizontal bonds are not ordered. As a result, the lattice strings are macroscopically flat. In terms of the height variables the order parameter is local and is given by $\left\langle\exp \left(i \pi \eta_{l}^{y}\right)\left(\eta_{l+1}^{y}-\eta_{l}^{y}\right)\right\rangle .^{14}$
TABLE I. A schematic representation of the different phases.

\begin{tabular}{|c|c|c|}
\hline Phase & String & Spin 1 \\
\hline I & & ++++++++ \\
\hline II & ......... & 00000000 \\
\hline III & $\therefore$ & +-+-+-+- \\
\hline IV & $\because$ & $+0-+0+0-+$ \\
\hline V & $\therefore$ & $-+0-00+-$ \\
\hline VI & & +-++-+-- \\
\hline VII & & $0+0++0+0 \quad 0$ \\
\hline VIII & $\ldots \bullet$ & $0+0+0+0+0$ \\
\hline IX & $\ldots \bullet^{\bullet} \ldots \cdots$ & $0+0+0-0+0$ \\
\hline $\mathrm{X}$ & $\ldots \cdots \ldots \cdots$ & $0+0-0+0-0$ \\
\hline
\end{tabular}

There are two new rough phases, again occurring when $L_{22} / 2-2 L_{21}$ is sufficiently large and positive. Region VII is a rough slanted phase: up steps are diluted by horizontal bonds with positional disorder. The average angle of the string is smaller than $45^{\circ}$ and the deviations from the average are again logarithmic. Phase IX is a rough phase where the even bonds are horizontal and the odd bonds are diagonal with up-down disorder. On average, the string is horizontal.

The results summarized in the phase diagram give a clear answer to whether and how quantum-domain walls can "melt"11: they can roughen either via a conventional Kosterlitz-Thouless transition (from phase II to IV), a firstorder KDP transition (phase I and IV), ${ }^{14}$ or a PokrovskyTalapov transition (phase I to VII). Because of the large hopping probability in the cuprates the parameter region of interest is $J$ and $D$ small, $J<0$. The relevant point is that domain walls come in two varieties: classically ordered and quantum rough. Such rough walls in our view are the building blocks of the conjectured domain-wall fluid and their low-lying collective excitations may be responsible for anomalous features of the metallic state. They give a natural explanation of the crossover scale proportional to the temperature as observed in the imaginary part of the susceptibility. ${ }^{10}$ The structural deformation of the LTT phase will suppress the diagonal segments, leading to an effective increase of the $K$ parameter. Therefore we envision that the formation of the stripe ordering in the La compounds ${ }^{2}$ is related to a transition from phase IV to phase II. At the transition meandering fluctuations become gapped. However, one should note that even in the most rough phase (phase IV) the meandering fluctuations are only logarithmic at low temperatures, and therefore the domain walls will be very sus- 
ceptible to wall-wall interactions and long-range Coulomb forces, which will promote ordering. ${ }^{17}$ Reconnection effects, ${ }^{13}$ however, will favor a fluid phase and compete with the above ordering mechanisms. Other crucial questions that need to be addressed is whether the domain wall scenario is compatible with the existence of fermionlike excitations, as seen, e.g., in photoemission experiments and in studies of the
$t-J$ model, and how exchange with free holes can be incorporated and affects the behavior.

H.E. is supported by the Stichting voor Fundamenteel Onderzoek der Materie (FOM), which is financially supported by the Nederlandse Organisatie voor Wetenschappelijk Onderzoek (NWO), and J.Z. acknowledges support by the Dutch Royal Academy of Sciences (KNAW).
${ }^{1}$ V. J. Emery and S. Kivelson, Phys. Rev. Lett. 74, 3253 (1995).

${ }^{2}$ J. M. Tranquada, B. J. Sternlieb, J. D. Axe, Y. Nakamura, and S. Uchida, Nature 375, 561 (1995).

${ }^{3}$ J. Zaanen and O. Gunnarson, Phys. Rev. B 40, 7391 (1989); D. Poilblanc and T. M. Rice, ibid. 39, 9749 (1989); H. J. Schulz, Phys. Rev. Lett. 64, 1445 (1990); M. Kato et al., J. Phys. Soc. Jpn. 59, 1047 (1990); M. Inui and P. B. Littlewood, Phys. Rev. B 44, 4415 (1991); J. A. Vergés, F. Guinea, and E. Louis, ibid. 46, 3562 (1992).

${ }^{4}$ P. Prelovšek and X. Zotos, Phys. Rev. B 47, 5984 (1993); T. Giamarchi and C. Lhuillier, ibid. 43, 12943 (1991); G. An and J. M. J. van Leeuwen, ibid. 44, 9410 (1991); H. J. M. van Bemmel et al., Phys. Rev. Lett. 72, 2442 (1994).

${ }^{5}$ U. Löw, V. J. Emery, K. Fabricius, and S. A. Kivelson, Phys. Rev. Lett. 72, 1918 (1994).

${ }^{6}$ J. Zaanen and P. B. Littlewood, Phys. Rev. B 50, 7222 (1994).

${ }^{7}$ J. M. Tranquada, D. J. Buttrey, V. Sachan, and J. E. Lorenzo, Phys. Rev. Lett. 73, 1003 (1994).

${ }^{8}$ S.-W. Cheong et al., Phys. Rev. Lett. 67, 1791 (1991); G. Shirane, R. J. Birgeneau, Y. Endoh, and M. A. Kastner, Physica B 197, 158 (1994); T. E. Mason, G. Aeppli, and H. A. Mook, Phys. Rev. Lett. 68, 1414 (1992); S. M. Hayden et al., ibid. 66,
821 (1991); B. Keimer et al., Phys. Rev. Lett. 67, 1930 (1991).

${ }^{9}$ V. Barzykin, D. Pines, and D. Thelen, Phys. Rev. B 50, 16052 (1994).

${ }^{10}$ J. Zaanen, M. L. Horbach, and W. van Saarloos, Phys. Rev. B 53, 8671 (1996).

${ }^{11}$ H. E. Viertiö and T. M. Rice, J. Phys. Condens. Matter 6, 7091 (1994).

${ }^{12}$ More generally, it is natural to take the hopping amplitudes of the last two configurations in Fig. 2(a) different.

${ }^{13} \mathrm{An}$ interesting and possibly important extension of (1) is to include terms describing self-avoidance and/or reconnection processes, the latter leading to string loops splitting off.

${ }^{14}$ M. den Nijs and K. Rommelse, Phys. Rev. B 40, 4709 (1989).

${ }^{15}$ H. Eskes, R. Grimberg, W. van Saarloos, and J. Zaanen (unpublished).

${ }^{16}$ Note that, while it was argued by D. S. Fisher and J. D. Weeks, Phys. Rev. Lett. 50, 1077 (1983), that quantum fluctuations cannot drive a two-dimensional interface rough at $T=0$, our results show that this does not extend to one-dimensional interfaces, i.e., strings.

${ }^{17}$ S. N. Coopersmith et al., Phys. Rev. B 25, 349 (1982). 